\title{
Site percolation thresholds on triangular lattice with complex neighbourhoods
}

\author{
Krzysztof Malarz* \\ AGH University of Science and Technology, Faculty of Physics and \\ Applied Computer Science, al. Mickiewicza 30, 30-059 Kraków, Poland
}

(Dated: October 24, 2020)

\begin{abstract}
We determine thresholds $p_{c}$ for random site percolation on a triangular lattice for neighbourhoods containing nearest $(\mathrm{NN})$, next-nearest $(2 \mathrm{NN})$, next-next-nearest $(3 \mathrm{NN})$, next-nextnext-nearest $(4 \mathrm{NN})$ and next-next-next-next-nearest $(5 \mathrm{NN})$ neighbours, and their combinations forming regular hexagons $(3 \mathrm{NN}+2 \mathrm{NN}+\mathrm{NN}, \quad 5 \mathrm{NN}+4 \mathrm{NN}+\mathrm{NN}, \quad 5 \mathrm{NN}+4 \mathrm{NN}+3 \mathrm{NN}+2 \mathrm{NN}$, $5 \mathrm{NN}+4 \mathrm{NN}+3 \mathrm{NN}+2 \mathrm{NN}+\mathrm{NN})$. We use a fast Monte Carlo algorithm, by Newman and Ziff [M. E. J. Newman and R. M. Ziff, Physical Review E 64, 016706 (2001)], for obtaining the dependence of the largest cluster size on occupation probability. The method is combined with a method, by Bastas et al. [N. Bastas, K. Kosmidis, P. Giazitzidis, and M. Maragakis, Physical Review E 90, 062101 (2014)], of estimating thresholds from low statistics data. The estimated values of percolation thresholds are $p_{c}(4 \mathrm{NN})=0.192410(43), p_{c}(3 \mathrm{NN}+2 \mathrm{NN})=0.232008(38)$, $p_{c}(5 \mathrm{NN}+4 \mathrm{NN})=0.140286(5), \quad p_{c}(3 \mathrm{NN}+2 \mathrm{NN}+\mathrm{NN})=0.215484(19), \quad p_{c}(5 \mathrm{NN}+4 \mathrm{NN}+\mathrm{NN})=$ $0.131792(58), \quad p_{c}(5 \mathrm{NN}+4 \mathrm{NN}+3 \mathrm{NN}+2 \mathrm{NN})=0.117579(41), \quad p_{c}(5 \mathrm{NN}+4 \mathrm{NN}+3 \mathrm{NN}+2 \mathrm{NN}+\mathrm{NN})=$ $0.115847(21)$. The method is tested on the standard case of site percolation on triangular lattice, where $p_{c}(\mathrm{NN})=p_{c}(2 \mathrm{NN})=p_{c}(3 \mathrm{NN})=p_{c}(5 \mathrm{NN})=\frac{1}{2}$ is recovered with five digits accuracy $p_{c}(\mathrm{NN})=0.500029(46)$ by averaging over one thousand lattice realisations only.
\end{abstract}

Keywords: sites percolation; triangular lattice; complex neighbourhoods; Newman-Ziff algorithm; Bastas et al. method; finite size scaling hypothesis

\section{INTRODUCTION}

The percolation theory $[1,2]$ - introduced in the middle 50's of the twentieth century [3, 4] - was recently applied in various fields of science ranging from agriculture [5] via studies of polymer composites [6], materials science [7], oil and gas exploration [8], quantifying urban areas [9] to transportation networks [10] (see Ref. 11 for review).

Usually, one assumes that the system percolates, when a cluster of occupied neighbouring sites spans between borders of the system. This happens when the occupation probability $p$ is greater than or equal to the percolation threshold $p_{c}$. The value $p_{c}$ is uniquely defined in the limit of infinite system size. The value of $p_{c}$ depends on network topology as well as on the sites' neighbourhood. By a site neighbourhood we mean a geometrical zone consisting of $z$ sites near the considered site. The sites may lie in the first, second, etc., coordinations zones. Percolation thresholds are known for many regular lattices blue in $d$-dimensional spaces (with $d$ up to 13) and for complex networks. One can find a list of known percolation thresholds in Ref. 12 and references therein.

In most cases only sites in the first coordination zone are included to site's neighbourhood. There are some exceptions - including seminal Domb and Dalton [13] paper - where people consider neighbourhoods consisting of several coordination zones, i.e. next-nearest neighbours, next-next-nearest neighbours, etc on hypercubic $[14,15]$, cubic $[16,17]$ or square [18-21] lattices. Much

\footnotetext{
* ORCID: 0000-0001-9980-0363; malarz@agh.edu.pl
}

less is know on percolation threshold values for complex neighbourhoods on other low-dimensional lattices - except of rough (up to $10^{-3}$ accuracy) threshold estimations for compact neighbourhoods on Archimedean lattices [22].

In this paper we try to fill this gap by estimating values of the percolation thresholds for several complex neighbourhoods on the triangular lattice. To that end we use a fast algorithm for percolation by Newman and Ziff [23] and a low sampling technique by Bastas et al. [24]. We determine percolation thresholds for random site percolation with several neighbourhoods containing the nearest neighbours $(\mathrm{NN})$, the next-nearest neighbours $(2 \mathrm{NN})$, the next-next-nearest neighbours $(3 \mathrm{NN})$, the next-nextnext-nearest neighbours $(4 \mathrm{NN})$ and the next-next-nextnext-nearest neighbours $(5 \mathrm{NN})$. All considered cases are schematically sketched in Fig. 1.

Percolation thresholds for lattices with complex neighbourhoods have been very recently successfully applied for many problems on square $[5,25,26]$ and cubic [2732] lattices. We believe that the results presented in this paper can also be applied to practical problems. For instance, the site-bond percolation in square, triangular, and honeycomb lattices [5] may be used to predict the minimal pathogen susceptibility to prevent the propagation of Phytophthora zoospores on Mexican chilli plantations. The $p_{c}$ values obtained in this work may also be helpful in searching for universal formulas for percolation thresholds in the spirit of recent attempts by Xun and Ziff [14]. 
(a) NN

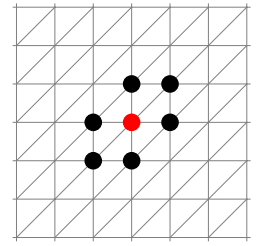

f) $3 \mathrm{NN}+2 \mathrm{NN}$

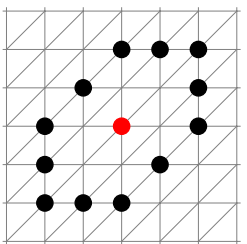

(b) $2 \mathrm{NN}$

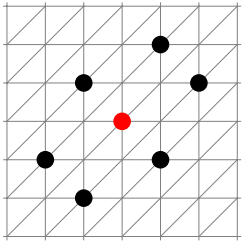

(c) $3 \mathrm{NN}$

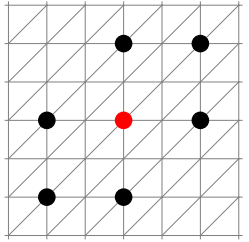

(d) $4 \mathrm{NN}$

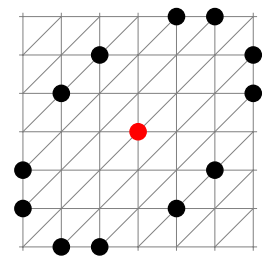

(h) $3 \mathrm{NN}$

(g) $5 \mathrm{NN}+4 \mathrm{NN}$

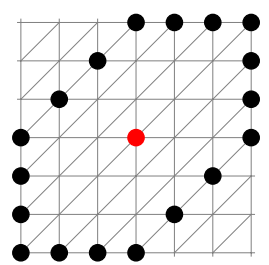

$+2 \mathrm{NN}+\mathrm{NN}$

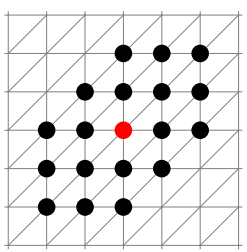

(i) $5 \mathrm{NN}$ $+4 \mathrm{NN}+\mathrm{NN}$

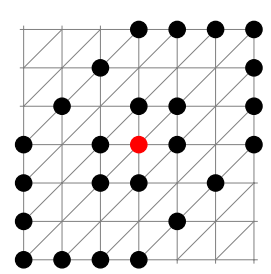

(j) $5 \mathrm{NN}+4 \mathrm{NN}$

$+3 \mathrm{NN}+2 \mathrm{NN}$

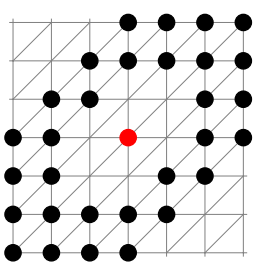

(e) $5 \mathrm{NN}$

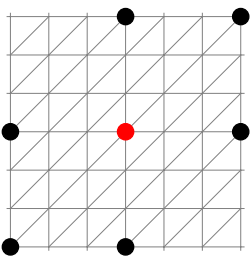

(k) $5 \mathrm{NN}+4 \mathrm{NN}$

$+3 \mathrm{NN}+2 \mathrm{NN}+\mathrm{NN}$

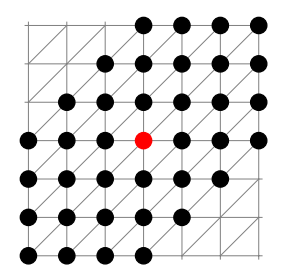

FIG. 1: Neighbourhoods containing (a) the nearest, (b) the next-nearest, (c) the next-next-nearest, (d) the next-next-next-nearest and (e) the next-next-next-next-nearest neighbours (and some of their combinations (f)-(k)) on triangular lattice.

\section{METHODS}

\section{A. Newman-Ziff method}

The idea behind the algorithm by Newman and Ziff [23] is based on the observation that some quantities can be calculated in the $(n, N)$ ensemble easier than in the $(p, N)$ ensemble. $N$ stands for the size of the system, $n$ for the number of occupied sites (or bonds) and $p$ for site (or bond) occupation probability. The relation between the two ensembles is similar to the relation between the $G(n, N)[33,34]$ and $G(p, N)[35]$ ensembles known from the construction of classical random graphs. In thermodynamic limit $(N \rightarrow \infty)$ these two approaches give the same results for $p=n / N$. The Authors [23] give several examples of quantities $\overline{\mathcal{A}}(n, N)$ which can be quickly computed in the $(n, N)$ ensemble by a recursive method. The algorithm by Newman and Ziff [23] is based on a recursive construction of states with $(n+1)$ occupied sites (or bonds) from states with $n$ occupied sites (or bonds). In a single step one adds a single site (or bond) and one applies union/find algorithm. The algorithm is very efficient.

Once the quantity $\overline{\mathcal{A}}(n, N)$ is determined for $n=$ $1,2, \cdots, N$, one can also reconstruct its counterpart in the $(p, N)$ ensemble by the following equation:

$$
\mathcal{A}(p ; N)=\sum_{n=1}^{N} \overline{\mathcal{A}}(n ; N) \mathcal{B}(n ; N, p),
$$

where

$$
\mathcal{B}(n ; N, p)=\left(\begin{array}{c}
N \\
n
\end{array}\right) p^{n}(1-p)^{N-n}
$$

For large $N$ and for $n \sim \mathcal{O}(N)$ one can approximate the Bernoulli distribution function by the Gauss curve:

$$
\mathcal{G}(n ; \mu, \sigma)=\frac{1}{\sqrt{2 \pi \sigma^{2}}} \exp \left(-\frac{(n-\mu)^{2}}{2 \sigma^{2}}\right),
$$

with the expected value $\mu=p N$ and variance $\sigma^{2}=p(1-$ p) $N$.

\section{B. Bastas et al. method}

The algorithm by Bastas et al. [24] relies on the scaling hypothesis $[36,37]$ which states that in the vicinity of a phase transition, many observables obey the following scaling law

$$
\mathcal{A}(p ; L)=L^{-x} \cdot \mathcal{F}\left(\left(p-p_{c}\right) L^{1 / \nu}\right)
$$

where $x$ and $\nu$ are some characteristic exponents, $L$ is the linear dimension of the system $\left(L \sim N^{1 / d}\right)$ and $\mathcal{F}$ is a universal scaling function $[1$, p. 71]. The product $\mathcal{A}(p ; L)$. $L^{x}$ is equal to $\mathcal{F}(0)$ for $p=p_{c}$ and thus it does not depend on the linear system size $L$. Therefore the curves $L^{x}$. $\mathcal{A}(p ; L)$ plotted for various values of $L$ should intercept in one point exactly at $p=p_{c}$. Instead of searching this crossing point the idea is to minimise the pairwise difference

$$
\Lambda(p, x)=\sum_{i \neq j}\left[\mathcal{H}\left(p ; L_{i}\right)-\mathcal{H}\left(p ; L_{j}\right)\right]^{2},
$$

where $\mathcal{H}(p ; L)$ is either $L^{x} \cdot \mathcal{A}(p ; L)$ [38] or $\mathcal{H}(p ; L)=$ $L^{x} \cdot \mathcal{A}(p ; L)+1 /\left(L^{x} \cdot \mathcal{A}(p ; L)\right)$ [24] and $i, j$ enumerate available system sizes $L$. 
The minimisation of $\Lambda(x, p)$ may be reduced to a single-value function $\lambda(p)$ minimisation for any observable $\mathcal{A}$ which does not require scaling along the $\mathcal{A}$ axis by a factor $L^{x}$ in order to achieve statistical invariance of the shape $\mathcal{A}(p ; L)$ for various values of $L$. Such a situation occurs for instance when one chooses the (top-bottom) wrapping probability function as $\mathcal{A}$ [16]. A similar reduction may be achieved also for any observable $\mathcal{A}$ for which the value of the exponent $x$ is known (note, that for wrapping probability function the scaling exponent is just $x=0$ ). An example of such an observable $\mathcal{A}$ is the probability that a randomly selected site belongs to the largest cluster

$$
\mathcal{P}_{\max }=\mathcal{S}_{\max } / N
$$

where $\mathcal{S}_{\max }$ is the size of the largest cluster (i.e. the number of sites which belong to it) and $N=L^{2}$. For $\mathcal{P}_{\max }$ the scaling exponent $x=\beta / \nu$

$$
\mathcal{P}_{\max }(p ; L)=L^{-\beta / \nu} \cdot \mathcal{F}\left(\left(p-p_{c}\right) L^{1 / \nu}\right)
$$

with exponents $\beta=\frac{5}{36}$ and $\nu=\frac{4}{3}$ [1, p. 54].

Here, to estimate the percolation thresholds $\hat{p}_{c}$ we minimise function

$$
\lambda(p)=\sum_{i \neq j}\left[\mathcal{H}\left(p ; L_{i}\right)-\mathcal{H}\left(p ; L_{j}\right)\right]^{2}
$$

with $\mathcal{H}(p ; L)=L^{\beta / \nu} \cdot \mathcal{P}_{\max }(p ; L)+1 /\left[L^{\beta / \nu} \cdot \mathcal{P}_{\max }(p ; L)\right]$.

\section{RESULTS}

In Fig. 2 we show the charts representing the dependence of $\left\langle\mathcal{P}_{\max }(p ; L)\right\rangle \cdot L^{\beta / \nu}$ on the sites occupation probabilities $p$ for various neighbourhoods and various linear system sizes $L=64,128,256,512,1024$, and 2048. The brackets denote averaging over $R=10^{3}$ independent simulations. The abscissas of the points where curves intercept estimate the percolation thresholds $\hat{p}_{c}$.

Unfortunately, each pair of curves intercept in different points. In contrast, the curves representing the dependence $\lambda(p)$ have clearly visible minima (see Fig. 3). The abscissa of this minimum estimates the percolation threshold $\hat{p}_{c}$.

Due to finite size effect, the obtained values of percolation thresholds $\hat{p}_{c}$ depends on system sizes used for $\lambda(p)$ calculations. The curves presented in Fig. 3 were obtained with summation in Eq. (8) over all six system sizes $L$ presented in Fig. 2. The summation over only five (from $L=64$ to $L=1024$ ) or four (from $L=64$ to $L=512$ ) terms in Eq. (8) results in changes of $\lambda(p)$ curves and position of their minima as for example (for $\mathrm{NN})$ presented in Fig. 4a. The obtained values of $\hat{p}_{c}(L)$ are marked as dots in Fig. 4b. According to finite size scaling prediction $[1$, p. 77$]$

$$
\hat{p}_{c}(L)=p_{c}+a \cdot L^{-1 / \nu},
$$

TABLE I: Random site triangular lattice percolation thresholds estimations $\hat{p}_{c}$ for various complex neighbourhoods. The middle column indicates the total number $z$ of sites forming the neighbourhood.

\begin{tabular}{rll}
\hline \hline neighbourhood & $z$ & $p_{c}$ \\
$\mathrm{NN}$ & 6 & $0.500029(46)$ \\
$2 \mathrm{NN}$ & 6 & $p_{c}(\mathrm{NN})$ \\
$3 \mathrm{NN}$ & 6 & $p_{c}(\mathrm{NN})$ \\
$4 \mathrm{NN}$ & 12 & $0.192410(43)$ \\
$5 \mathrm{NN}$ & 6 & $p_{c}(\mathrm{NN})$ \\
$3 \mathrm{NN}+2 \mathrm{NN}$ & 12 & $0.232008(38)$ \\
$5 \mathrm{NN}+4 \mathrm{NN}$ & 18 & $0.140286(5)$ \\
$3 \mathrm{NN}+2 \mathrm{NN}+\mathrm{NN}$ & 18 & $0.215484(19)$ \\
$5 \mathrm{NN}+4 \mathrm{NN}+\mathrm{NN}$ & 24 & $0.131792(58)$ \\
$5 \mathrm{NN}+4 \mathrm{NN}+3 \mathrm{NN}+2 \mathrm{NN}$ & 30 & $0.117579(41)$ \\
$5 \mathrm{NN}+4 \mathrm{NN}+3 \mathrm{NN}+2 \mathrm{NN}+\mathrm{NN}$ & 36 & $0.115847(21)$ \\
\hline \hline
\end{tabular}

where $p_{c}$ is the percolation threshold for infinitely large system. The solid line in Fig. $4 \mathrm{~b}$ is the least squares method linear fit of $\hat{p}_{c}$ versus $L^{-1 / \nu}$, and uncertainty of estimation the fit parameter predicts the uncertainty $u\left(p_{c}\right)$ of percolation threshold. The obtained percolation thresholds $p_{c}$ (for $L \rightarrow \infty$ ) together with their uncertainties are gathered in Table I.

\section{CONCLUSIONS}

In this paper we estimated percolation thresholds $p_{c}$ for random site triangular lattice percolation and for neighbourhoods containing NN, $2 \mathrm{NN}, 3 \mathrm{NN}, 4 \mathrm{NN}$ and $5 \mathrm{NN}$. The estimated values of percolation thresholds are collected in Table I. As a triangular lattice with $2 \mathrm{NN}$ (3NN, $5 \mathrm{NN})$ neighbours may be mapped onto independent interpenetrated triangular lattices but with $\sqrt{3}(2$, $3)$ times larger lattice constant the percolation thresholds $p_{c}(2 \mathrm{NN})=p_{c}(3 \mathrm{NN})=p_{c}(5 \mathrm{NN})$ are exactly equal to $p_{c}(\mathrm{NN})$.

We adopted the algorithm by Newman and Ziff [23] and the technique by Bastas et al. [24] to estimate these values.

In the algorithm by Newman and Ziff [23] we replaced the Bernoulli probability distribution function with a Gaussian $\mathcal{B}(n ; N, p) \approx \mathcal{G}(n ; \mu, \sigma)$ with $\mu=p N$ and $\sigma=\sqrt{p(1-p) N}$. Based on hypothesis of critical exponents universality we simplified the Bastas et al. [24] algorithm by reducing the problem of minimisation of a multidimensional function $\Lambda(p, x)$ to a problem of minimisation of a single-valued function $\lambda(p)$ by using the fact that one knows the exact value of the critical exponent $x=\beta / \nu=\frac{5}{48}$ for $\mathcal{P}_{\max }$ in two dimensions.

The obtained results improve the earlier estimations of percolation thresholds $p_{c}=0.215$ for $3 \mathrm{NN}+2 \mathrm{NN}+\mathrm{NN}$ neighbourhood and $p_{c}=0.115$ for 
(a) NN

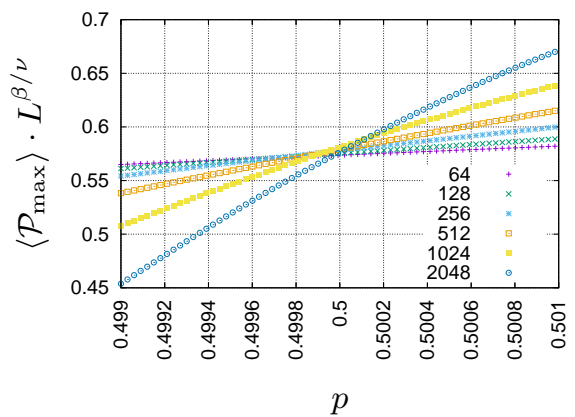

(c) $3 \mathrm{NN}+2 \mathrm{NN}$

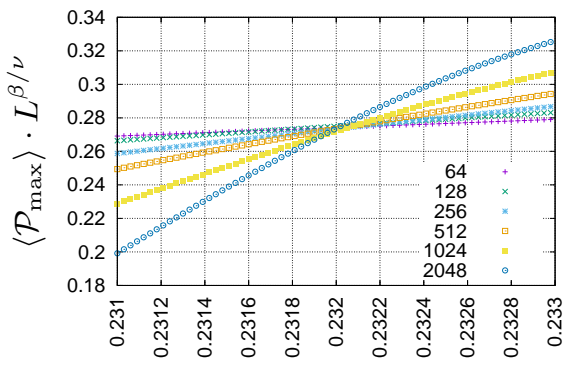

$p$

(f) $5 \mathrm{NN}+4 \mathrm{NN}+\mathrm{NN}$

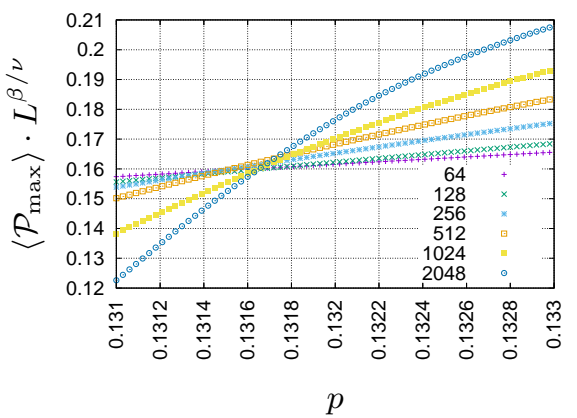

(d) $5 \mathrm{NN}+4 \mathrm{NN}$

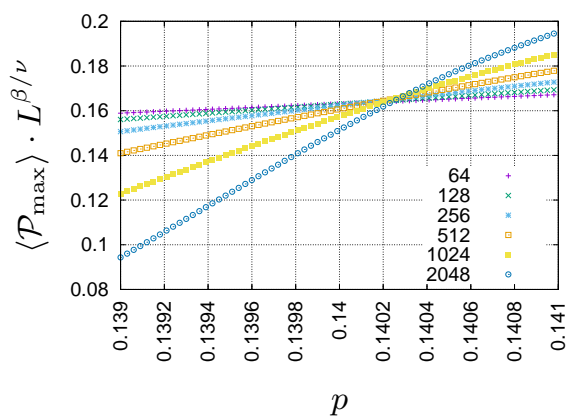

(g) $5 \mathrm{NN}+4 \mathrm{NN}+3 \mathrm{NN}+2 \mathrm{NN}$

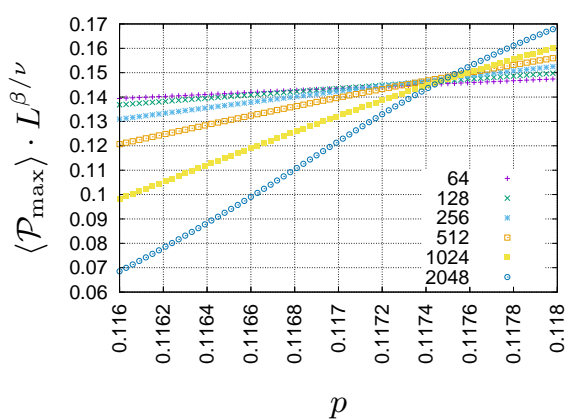

(b) $4 \mathrm{NN}$

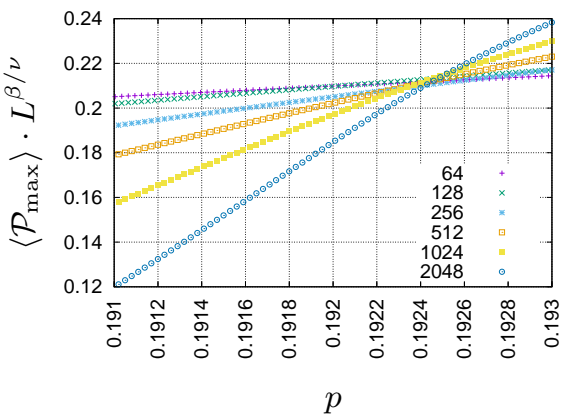

(e) $3 \mathrm{NN}+2 \mathrm{NN}+\mathrm{NN}$ (h) $5 \mathrm{NN}+4 \mathrm{NN}+3 \mathrm{NN}+2 \mathrm{NN}+\mathrm{NN}$

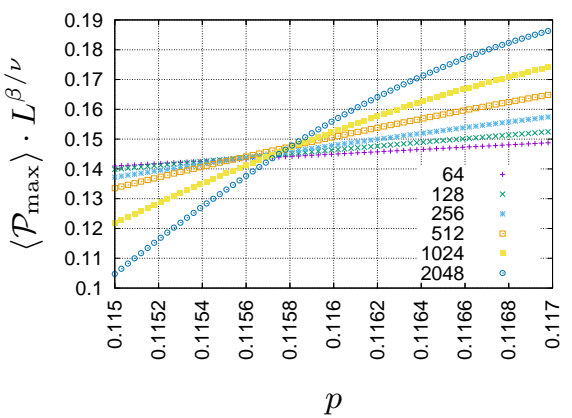

FIG. 2: Dependencies of $\left\langle\mathcal{P}_{\max }(p ; L)\right\rangle \cdot L^{\beta / \nu}$ on occupation probability $p$ for $L=64,128,256,512,1024,2048$ and for various neighbourhoods: (a) $\mathrm{NN}$, (b) $4 \mathrm{NN}$, (c) $3 \mathrm{NN}+2 \mathrm{NN}$, (d) $5 \mathrm{NN}+4 \mathrm{NN}$, (e) $3 \mathrm{NN}+2 \mathrm{NN}+\mathrm{NN}$, (f) $5 \mathrm{NN}+4 \mathrm{NN}+\mathrm{NN},(\mathrm{g}) 5 \mathrm{NN}+4 \mathrm{NN}+3 \mathrm{NN}+2 \mathrm{NN},(\mathrm{h}) 5 \mathrm{NN}+4 \mathrm{NN}+3 \mathrm{NN}+2 \mathrm{NN}+\mathrm{NN}$.

$5 \mathrm{NN}+4 \mathrm{NN}+3 \mathrm{NN}+2 \mathrm{NN}+\mathrm{NN}$ neighbourhood and they agree nicely with first three digits available in Ref. 22.
The Domb and Dalton [13] result published over five decades ago agrees only qualitatively (0.225 vs. $0.215484(19))$.
[1] D. Stauffer and A. Aharony, Introduction to Percolation Theory, 2nd ed. (Taylor and Francis, London, 1994).

[2] J. Wierman, Percolation theory, in Wiley StatsRef: Statistics Reference Online (American Cancer Society, 2014) pp. 1-9.

[3] S. R. Broadbent and J. M. Hammersley, Percolation processes: I. Crystals and mazes, Mathematical Proceedings of the Cambridge Philosophical Society 53, 629-641 (1957).

[4] J. M. Hammersley, Percolation processes: II. The connective constant, Mathematical Proceedings of the Cam- bridge Philosophical Society 53, 642-645 (1957).

[5] J. E. Ramirez, C. Pajares, M. Martinez, I, R. Rodriguez Fernandez, E. Molina-Gayosso, J. LozadaLechuga, and A. Fernandez Tellez, Site-bond percolation solution to preventing the propagation of Phytophthora zoospores on plantations, Physical Review E 101, 032301 (2020).

[6] Q. Zhang, B.-Y. Zhang, B.-H. Guo, Z.-X. Guo, and J. Yu, High-temperature polymer conductors with self-assembled conductive pathways, Composites Part B-Engineering 192, 107989 (2020). 
(a) NN

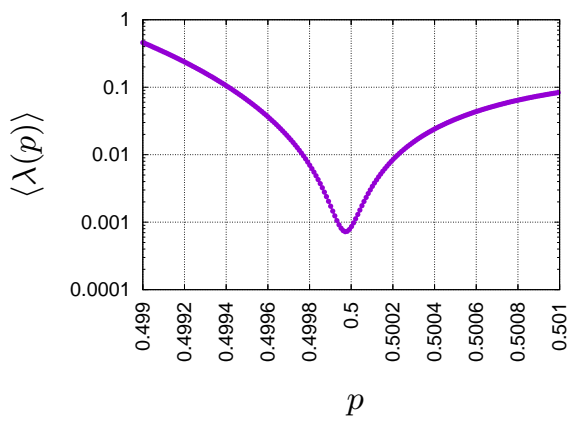

(d) $5 \mathrm{NN}+4 \mathrm{NN}$

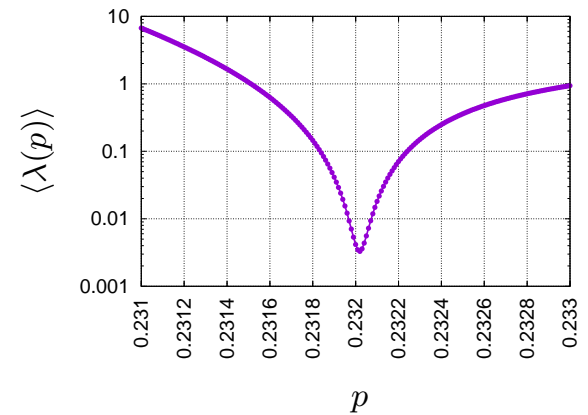

(f) $5 \mathrm{NN}+4 \mathrm{NN}+\mathrm{NN}$

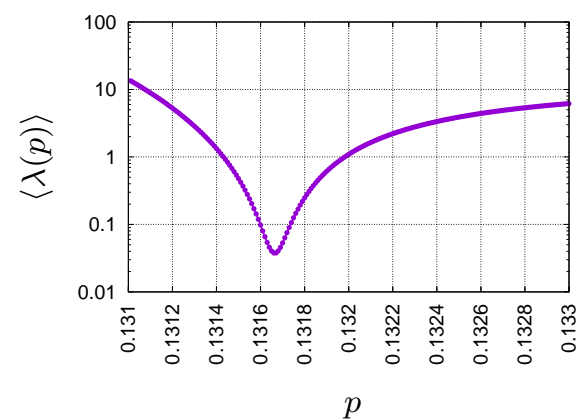

(b) $4 \mathrm{NN}$

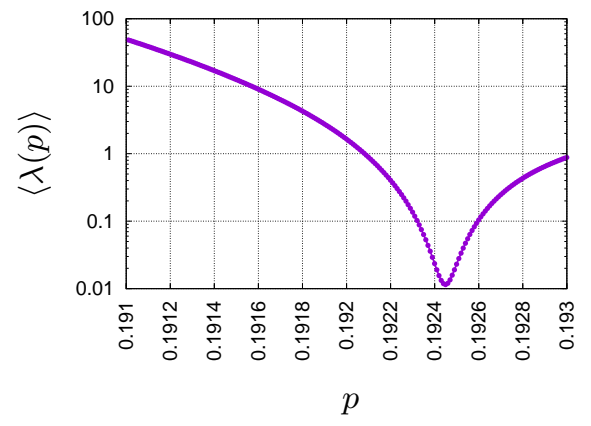

(e) $3 \mathrm{NN}+2 \mathrm{NN}+\mathrm{NN}$

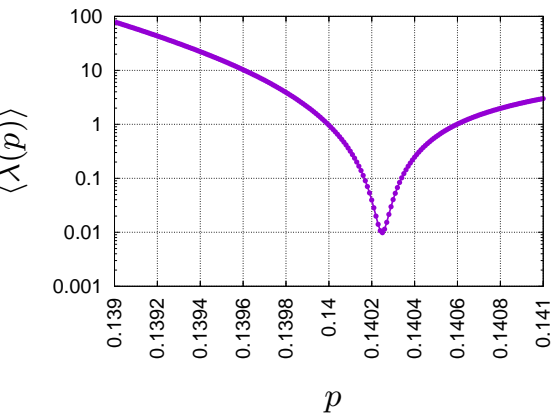

(g) $5 \mathrm{NN}+4 \mathrm{NN}+3 \mathrm{NN}+2 \mathrm{NN}$

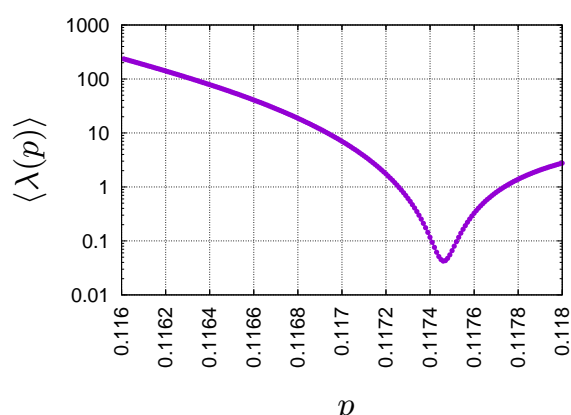

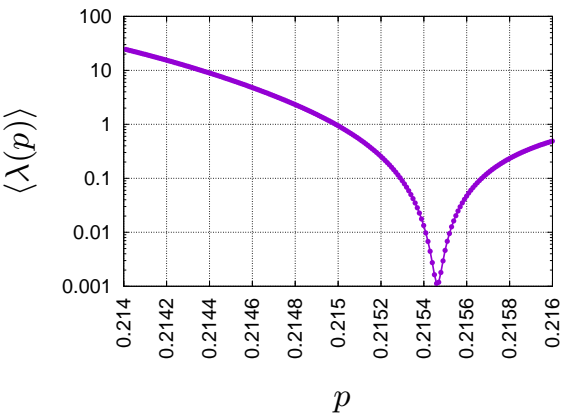

(h) $5 \mathrm{NN}+4 \mathrm{NN}+3 \mathrm{NN}+2 \mathrm{NN}+\mathrm{NN}$

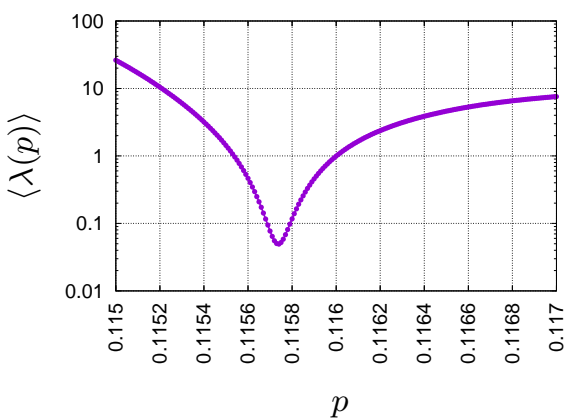

FIG. 3: Dependencies of $\langle\lambda(p)\rangle$ on occupation probability $p$ for various neighbourhoods: (a) NN, (b) 4NN, (c) $3 \mathrm{NN}+2 \mathrm{NN}$, (d) $5 \mathrm{NN}+4 \mathrm{NN}$, (e) $3 \mathrm{NN}+2 \mathrm{NN}+\mathrm{NN}$, (f) $5 \mathrm{NN}+4 \mathrm{NN}+\mathrm{NN},(\mathrm{g}) 5 \mathrm{NN}+4 \mathrm{NN}+3 \mathrm{NN}+2 \mathrm{NN},(\mathrm{h})$ $5 \mathrm{NN}+4 \mathrm{NN}+3 \mathrm{NN}+2 \mathrm{NN}+\mathrm{NN}$. The minima give estimates of the percolation thresholds $\hat{p}_{c}$ for all six system sizes $L=64,128,256,512,1024,2048$ used for summation in Eq. (8).

[7] L. Cheng, P. Yan, X. Yang, H. Zou, H. Yang, and H. Liang, High conductivity, percolation behavior and dielectric relaxation of hybrid ZIF-8/CNT composites, Journal of Alloys and Compounds 825, 154132 (2020).

[8] B. Ghanbarian, F. Liang, and H.-H. Liu, Modeling gas relative permeability in shales and tight porous rocks, Fuel 272, 117686 (2020).

[9] W. Cao, L. Dong, L. Wu, and Y. Liu, Quantifying urban areas with multi-source data based on percolation theory, Remote Sensing of Environment 241, 111730 (2020).

[10] S. Dong, A. Mostafizi, H. Wang, J. Gao, and X. Li, Measuring the topological robustness of transportation networks to disaster-induced failures: A percolation approach, Journal of Infrastructure Systems 26, 04020009 (2020).

[11] A. A. Saberi, Recent advances in percolation theory and its applications, Physics Reports 578, 1-32 (2015).
[12] en.wikipedia.org/wiki/percolation_threshold (2020).

[13] C. Domb and N. W. Dalton, Crystal statistics with longrange forces: I. The equivalent neighbour model, Proceedings of the Physical Society 89, 859-871 (1966).

[14] Z. Xun and R. M. Ziff, Precise bond percolation thresholds on several four-dimensional lattices, Physical Review Research 2, 013067 (2020).

[15] M. Kotwica, P. Gronek, and K. Malarz, Efficient space virtualisation for Hoshen-Kopelman algorithm, International Journal of Modern Physics C 30, 1950055 (2019).

[16] K. Malarz, Simple cubic random-site percolation thresholds for neighborhoods containing fourth-nearest neighbors, Physical Review E 91, 043301 (2015).

[17] Ł. Kurzawski and K. Malarz, Simple cubic random-site percolation thresholds for complex neighbourhoods, Reports on Mathematical Physics 70, 163-169 (2012).

[18] M. Gouker and F. Family, Evidence for classical critical 
(a) NN: $\langle\lambda(p)\rangle$

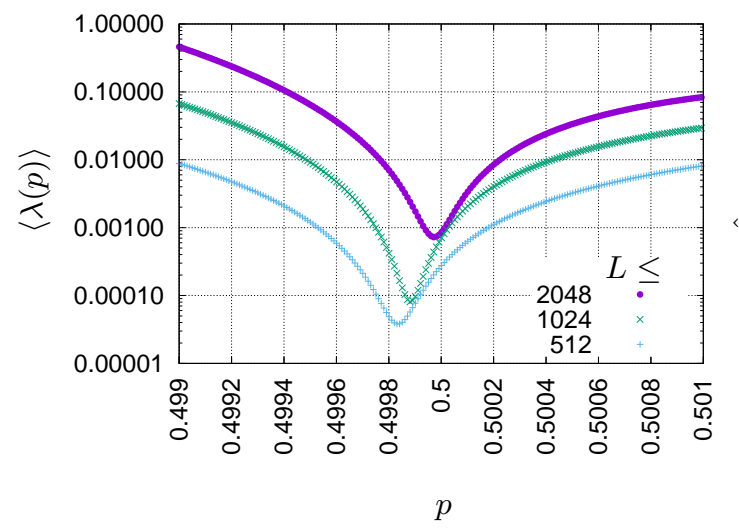

(b) NN: $\hat{p}_{c}$ vs. $L^{-1 / \nu}$

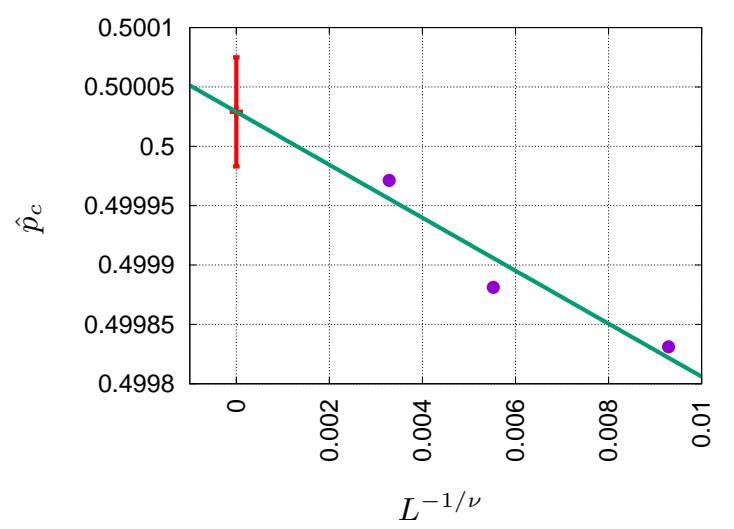

FIG. 4: The finite size scaling analysis for NN. (a) $\langle\lambda(p)\rangle$ for various number of summation terms in Eq. (8). (b) The least squares method fit for $\hat{p}_{c}(L)$ vs. $L^{-1 / \nu}$. The intersection of the linear fit with vertical axis $L^{-1 / \nu}=0$ predicts percolation threshold $p_{c}$ for infinitely large system.

behavior in long-range site percolation, Physical Review B 28, 1449-1452 (1983).

[19] M. Majewski and K. Malarz, Square lattice site percolation thresholds for complex neighbourhoods, Acta Physica Polonica B 38, 2191-2199 (2007).

[20] K. Malarz and S. Galam, Square-lattice site percolation at increasing ranges of neighbor bonds, Physical Review E 71, 016125 (2005).

[21] S. Galam and K. Malarz, Restoring site percolation on damaged square lattices, Physical Review E 72, 027103 (2005).

[22] C. d'Iribarne, M. Rasigni, and G. Rasigni, From lattice long-range percolation to the continuum one, Physics Letters A 263, 65-69 (1999).

[23] M. E. J. Newman and R. M. Ziff, Fast Monte Carlo algorithm for site or bond percolation, Physical Review E 64, 016706 (2001).

[24] N. Bastas, K. Kosmidis, P. Giazitzidis, and M. Maragakis, Method for estimating critical exponents in percolation processes with low sampling, Physical Review E 90, 062101 (2014).

[25] V. Negi and R. C. Picu, Elastic-plastic transition in stochastic heterogeneous materials: Size effect and triaxiality, Mechanics of Materials 120, 26-33 (2018).

[26] L. Keeney, C. Downing, M. Schmidt, M. E. Pemble, V. Nicolosi, and R. W. Whatmore, Direct atomic scale determination of magnetic ion partition in a room temperature multiferroic material, Scientific Reports 7, 1737 (2017).

[27] D. Soto-Gomez, L. Vazquez Juiz, P. Perez-Rodriguez, J. Eugenio Lopez-Periago, M. Paradelo, and J. Koestel, Percolation theory applied to soil tomography, Geoderma 357, 113959 (2020).

[28] A. Avella, A. M. Oles, and P. Horsch, Defect-induced orbital polarization and collapse of orbital order in doped vanadium perovskites, Physical Review Letters 122, 127206 (2019).
[29] W. W. Erikson, Thermal decomposition of ammonium perchlorate using Monte Carlo methods, Journal of Energetic Materials 37, 222-239 (2019).

[30] B. G. Ueland, N. H. Jo, A. Sapkota, W. Tian, M. Masters, H. Hodovanets, S. S. Downing, C. Schmidt, R. J. McQueeney, S. L. Bud'ko, A. Kreyssig, P. C. Canfield, and A. I. Goldman, Reduction of the ordered magnetic moment and its relationship to Kondo coherence in $\mathrm{Ce}_{1-x} \mathrm{La}_{x} \mathrm{Cu}_{2} \mathrm{Ge}_{2}$, Physical Review B 97, 165121 (2018).

[31] J. Jeong, K. J. Park, E.-J. Cho, H.-J. Noh, S. B. Kim, and H.-D. Kim, Electronic structure change of $\mathrm{NiS}_{2-x} \mathrm{Se}_{x}$ in the metal-insulator transition probed by X-ray absorption spectroscopy, Journal of the Korean Physical Society 72 , 111-115 (2018).

[32] T. Moench, P. Friederich, F. Holzmueller, B. Rutkowski, J. Benduhn, T. Strunk, C. Koerner, K. Vandewal, A. Czyrska-Filemonowicz, W. Wenzel, and K. Leo, Influence of meso and nanoscale structure on the properties of highly efficient small molecule solar cells, Advanced Energy Materials 6, 1501280 (2016).

[33] P. Erdős and A. Rényi, On random graphs. I, Publicationes Mathematicae 6, 290-297 (1959).

[34] P. Erdős and A. Rényi, On the evolution of random graphs, Publications of the Mathematical Institute of the Hungarian Academy of Sciences 5, 17-61 (1960).

[35] E. N. Gilbert, Random graphs, The Annals of Mathematical Statistics 30, 1141-1144 (1959).

[36] V. Privman, Finite-size scaling theory, in Finite size scaling and numerical simulation of statistical systems, edited by V. Privman (World Scientific, Singapore, 1990) pp. 1-98.

[37] D. P. Landau and K. Binder, A Guide to Monte Carlo Simulations in Statistical Physics, 2nd ed. (Cambridge UP, Cambridge, 2005).

[38] N. Bastas, K. Kosmidis, and P. Argyrakis, Explosive site percolation and finite-size hysteresis, Physical Review E 84, 066112 (2011). 